\title{
The Incidental Hematopoietic Evaluation - Microscopic Examination of Routine Orthopedic Surgical Specimens
}

Jesse S Bond and Candice C Black ${ }^{*}$

Department of Pathology, Dartmouth-Hitchcock Medical Center, One Medical Center Drive, Lebanon

"Corresponding author: Candice C Black, Department of Pathology, Dartmouth-Hitchcock Medical Center, One Medical Center Drive, NH 03756, Lebanon, Tel: 603-650-7211; Fax: 603-650-7214; E-mail: Candice.C.Black@hitchcock.org

Rec date: Mar 21, 2014, Acc date: June 04, 2014, Pub date: June 06, 2014

Copyright: () 2014 Bond SJ, et al. This is an open-access article distributed under the terms of the Creative Commons Attribution License, which permits unrestricted use, distribution, and reproduction in any medium, provided the original author and source are credited.

\begin{abstract}
Routine orthopedic specimens provide a unique opportunity to review the bone marrow of patients. The processing of these specimens differs at different hospitals and has been scrutinized lately due to the recent wave of cost consciousness in health care. We review examples of important unexpected findings in such cases, as well as the benefits of review, in light of studies showing the rarity of such findings.
\end{abstract}

Keywords: Orthopedic specimen; Specimen review; Histologic review; Pathology review; Marrow review

\section{Review on Orthopedic Surgical Specimens}

There is no practice that is universally accepted for the examination of orthopedic surgical tissues by pathology departments. The handling of routine orthopedic specimens is an issue that is treated differently at different institutions. Management can vary from submitting tissue sections for histology on every case, to macroscopic (gross) exam alone, or to throwing the tissue into waste receptacles in the operating room. The pathologic examination can serve as a quality assurance procedure, providing verification to the patient of the exact tissues removed and diagnosis of each tissue. The Joint Commission on Accreditation of Health Care Organizations allows the clinical staff to decide exceptions to the rule of routine pathologic examination of all surgical specimens, as long as there is an alternate process for verifying the removal of the tissues, and patient care is not compromised. In many cases the board of trustees and/or Board of Governors weigh in on this decision.

There has been an initiative by some groups to eliminate the microscopic (histologic) review of routine femoral head and total knee arthroplasty specimens in the pathology lab [1-6]. Increased cost to the patient is the main consideration with secondary concern about reimbursement from health care insurers. It has been noted, however, that if there is discordance between the clinical and pathologic diagnosis, then examination is warranted, however, this would not be possible for discarded tissues. In most cases, the surgeon may request pathologic exam on a case by case basis, if there is a pathologic fracture or other clinical indication. Abnormalities of the marrow cavity however are likely to be hidden from the surgeon's brief intraoperative macroscopic exam. Studies comparing pre-operative and postoperative (histologic) diagnoses usually distinguish those diagnoses as clinically actionable or discordant (treatment or change of treatment of the patient is required immediately) versus those diagnoses that are different (discrepant) but do not change the care of the patient in any immediate way. The distinction of the former from the latter has been hotly debated [7]. There has been concern that the diagnoses considered irrelevant to patient care by some (i.e. hemochromatosis, rheumatoid arthritis, atypical lymphoid aggregates) are in fact important though not immediately actionable. Studies show that discordant diagnosis rates are rare with ranges from $0.8 \%$ to $8.9 \%$ [1].

Kocher et al. [1] reviewed 1234 specimens of femoral head and knee and showed only one discordant case that resulted in a clinically (immediate) actionable diagnosis (0.1\%). The discrepant rate was 2.3 $\%$. They estimated that the cost per discrepant diagnosis was $\$ 4383$, and the cost per discordant diagnosis $\$ 122,728$. A more recent study [8] found a $2.1 \%$ discrepant rate with a cost estimate of $\$ 4983$ per case, nearly the same amount as Kocher et al. [1] No discordant cases were found in this study; however, there was less medical follow-up of cases, as this was focused mainly on medical economics. A separate retrospective analysis of 2144 cases, limited to shoulder arthroscopy [2], showed no discordant pathologic diagnoses when compared to the clinical diagnosis. These studies have concluded that it is not cost effective to examine surgical tissues histologically.

In some countries such as Australia, [9] arthroscopic femoral head specimens are routinely collected for allograft bone transplant banking. This necessitates a protocol of routine histologic exam of at least a sample of the bone, as well as serologic testing of patients for infection with cultures and in-depth history and physical exam. They found in a series of 6161 cases, $1.7 \%$ (105 cases) were rejected due solely to histologic findings, similar to cases rejected due to positive microbial cultures, and greater than those rejected due to HIV, syphilis and HTLV combined. Nineteen incidental hematopoietic malignancies were documented (mainly non-Hodgkin lymphoma and B-cell lymphoma). They estimate 1 in 770 neoplasms per femoral head-histologic examinations. The time for full disease manifestation in a few cases lagged for up to 4 years, but the patients were routed clinically to appropriate monitoring. Mackie et al. [9] note that examination of femoral head marrow is a decent screening technique compared with other accepted screenings. Mammography detects 6 malignancies per 1904 screenings and flexible sigmoidoscopy detects 5 in 489. A similar study of femoral head bone banking from the Netherlands [10] found similar numbers of low grade B-cell lymphomas (13 in 851 cases) that were rejected as well as 6 of 504 with atypical lymphoid aggregates, not rejected from the bank. Specifically following the atypical group, they found that one of the patients developed a B-cell lymphoma in a lymph node and required 
chemotherapy. Three years later, 3 of the "atypical" group were still being monitored by hematologists for atypia. They note that NHL is increasing, with the expectation of a $36.1 \%$ increase between 2000 and 2020.

Case reports and small series continue to show incidental hematopoietic disorders discovered during routine histologic examination of orthopedic specimens [6,11-13]. We recently reported a case of incidental mastocytosis in a 49 year old female undergoing an elective total hip arthroplasty for presumed osteoarthritis [6]. The patient had voiced complaints to her primary care doctor of severe, bilateral hip pain that had forced her to make ergonomic changes at the workplace. The mastocytsis was seen in the marrow of her arthritic joint tissues and would have been missed without microscopic examination.

A report of a 56 year old male with Non-Hodgkins lymphoma was made after examination of a routine hip arthroscopy specimen [11]. A lumbar bone marrow aspirate confirmed this diagnosis, as low grade follicular type. Review of pre-operative serologies showed a normal complete blood count with differential as well as a normal chest radiograph and chemistry panel. Eight months after the diagnosis however, the patient became leukopenic and neutropenic.

Other diagnoses found in arthritic bones include myeloproliferative disorder, Rheumatoid arthritis, gout, PVNS and hemochromatosis $[1,4]$. Incidental sarcoma was found in the hip specimen from a patient with a history pelvic radiation for cervical carcinoma. Further sectioning of the bone showed soft marrow with areas of red-brown necrosis. A second case showed incidental malignant fibrous histiocytoma (Undifferentiated pleomorphic sarcoma) in the setting of Paget disease. No tumor was macroscopically seen in either case [13]. Pui and Jergesen [14] report a 25 year old with arthroscopy for hip pain. The specimen was deemed to be arthritis during the OR gross exam and discarded, a cyst was curetted, packed and prosthesis was placed. One year later, lytic lesions formed leading to the clinical suspicion of peri-prosthetic infection. At re-operation the hardware was removed and a cement spacer was placed. Tissue was sent for histologic exam and the disease Langerhans cell histiocytosis (LCH) was diagnosed, which likely was the causative pathology for this patient's entire disease process. This is a disease that can require systemic medicine, and it can have implications of recurrence and multifocality.

Our current institutional policy for handling these specimens is macroscopic exam if osteoarthritis/degenerative joint disease is the only clinical diagnosis however the surgeon can request full review with histologic examination. If the macroscopic pathology exam shows findings concordant with the clinical diagnosis, no microscopic exam will be pursued. A preconceived bias that the disease is "just" arthritis however, can lead to the discarding of truly discordant tissues, as in the case of LCH. If there is a discrepant macroscopic finding, or at the pathologist's request following chart review, microscopy will be pursued. Strong communication between the orthopedic surgeon and the pathologist is an important link in making this an efficient system for catching discrepant diagnoses.

If the orthopedic surgeon is aware of the institutional policy for handling arthroplasty specimens then they will have an increased awareness for communicating other diagnostic considerations for the patients. This point was nicely illustrated in an editorial response by Clark and Bauer [15] in a discussion of the possible ways to handle these specimens in a cost efficient manner. They acknowledge that cost is an important concern; but that the histologic study of the tissue serves many purposes in addition to immediate patient care, including systemic disease care planning and specialized patient monitoring (i.e. rheumatology, hematology). The general practice we have adopted for femoral head and knee arthroplasty specimens includes a combination of their suggestions for improving cost efficiency without excluding pathologic examination entirely. While histology for all cases is the gold standard, some compromise must be made to keep costs down. We suggest that the surgeon considers any outlier symptoms, lab findings, or clinical indications when making the decision for histology.

As the pathology profession shifts evermore towards sub specialization, it becomes more important that we pay close attention to the portions of the routine specimens that are outside our respective specialties. Access to examination of orthopedic tissues, including routine marrow reviews, plays an import role in the education of pathology trainees. Trainee competence in the ability to perform unsupervised examination of these tissues must be documented for the Accreditation Council for Graduate Medical Education (ACGME).

The diagnostic yield of microscopic examination on arthroplasty specimens is low but it can result in a life changing diagnosis for the patient. The important component to selecting these specimens is to have good communication with the surgeons and a solid understanding of the specimen policies for all parties involved. In the rare case where the pathologist detects a subtle abnormality in the marrow, obtaining a hematologic consult may prove fruitful.

\section{References}

1. Kocher MS, Erens G, Thornhill TS, Ready JE (2000) Cost and effectiveness of routine pathological examination of operative specimens obtained during primary total hip and knee replacement in patients with osteoarthritis. J Bone Joint Surg Am 82-82A: 1531-5.

2. McClain RE, Hotari CD, Scribner CL, Detrisac DA (2008) The clinical value of histologic examination in shoulder arthroscopy. J Bone Joint Surg Am 90: 281-283.

3. Meding JB, Ritter MA, Jones NL, Keating EM, Faris PM (2000) Determining the necessity for routine pathologic examinations in uncomplicated total hip and total knee arthroplasties. J Arthroplasty 15: 69-71.

4. Pagnano MW, Forero JH, Scuderi GR, Harwin SF (1998) Is the routine examination of surgical specimens worthwhile in primary total knee arthroplasty? Clin Orthop Relat Res: 79-84.

5. Campbell ML, Gregory AM, Mauerhan DR (1997) Collection of surgical specimens in total joint arthroplasty. Is routine pathology cost effective? J Arthroplasty 12: 60-63.

6. Bond JS, Black CC, Kantor SP, Ornstein DL (2014) Systemic Mastocytosis Discovered Incidentally After an Elective Femoral Head Arthroplasty.

7. Palmer SH, Kocher MS, Erens G, Thronhill TS, Ready JE (2002) Routine Pathological Examination of Surgical Specimens from Patients Undergoing Total Hip and Knee Replacement. J Bone Joint Surg Am 84: 1082-1084.

8. Lin MM, Goldsmith JD, Resch SC, DeAngelis JP, Ramappa AJ (2012) Histologic examinations of arthroplasty specimens are not cost-effective: a retrospective cohort study. Clin Orthop Relat Res 470: 1452-1460.

9. Mackie KE, Zhou Z, Robbins P, Bulsara M, Zheng MH (2011) Histopathology of femoral head donations: a retrospective review of 6161 cases. J Bone Joint Surg Am 93: 1500-1509.

10. Zwitser EW, de Gast A, Basie MJ, van Kemenade FJ, van Royen BJ (2009) B-cell lymphoma in retrieved femoral heads: a long term follow up. BMC Musculoskelet Disord 10: 53. 
Citation: Bond JS, Black CC (2014) The Incidental Hematopoietic Evaluation - Microscopic Examination of Routine Orthopedic Surgical Specimens. J Blood Lymph 4: 122. doi:10.4172/2165-7831.1000122

Page 3 of 3

11. Lauder AJ, Cheatham SA, Garvin KL (2004) Unsuspected non-Hodgkin's lymphoma discovered with routine histopathology after elective total hip arthroplasty. J Arthroplasty 19: 1055-1060.

12. Arredondo J, Worland RL, Sinnenberg RJ Jr, Qureshi GD (1999) NonHodgkin's lymphoma as an unexpected diagnosis in a shoulder arthroplasty. J Arthroplasty 14: 108-111.

13. Billings SD, Wurtz LD, Tejada E, Henley JD (2000) Occult sarcoma of the femoral head in patients undergoing total hip arthroplasty. A report of two cases. J Bone Joint Surg Am 82-82A: 1536-1539.
14. Pui CM, Jergesen HE (2011) Femoral involvement by langerhans cell histiocytosis following total hip arthroplasty: a case report. J Bone Joint Surg Am 93.

15. Clark CR, Bauer T (2000) Routine pathological examination of operative specimens from primary total hip and total knee replacement: another look. J Bone Joint Surg Am 82-82A: 1529-1530. 